\title{
Detection of Parsec-Scale Circular Polarization with the VLBA
}

\author{
D. C. Homan, J. F. C. Wardle, R. Ojha, \& D. H. Roberts \\ Brandeis University, Waltham, MA 02254, USA
}

\begin{abstract}
Circular polarization has been detected in 3C 279 and 3C 84 at multiple epochs, using the VLBA at $15 \mathrm{GHz}$. This is the first detection of circular polarization in a compact radio source at VLBI resolution. In the case of $3 \mathrm{C} 279$ the polarization is most likely produced by the conversion of linear to circular polarization by low energy relativistic electrons in the emitting region. If this is so, the absence of Faraday rotation by the same electrons suggests that the jet consists of an electron-positron plasma.
\end{abstract}

The excellent sensitivity and uniformity of calibration of the VLBA makes it possible to detect circularly polarized signals at fractional levels less than $0.5 \%$ locally and $0.1 \%$ of the total flux, if the source is sufficiently strong. We have detected circular polarization in 3C 279 and 3C 84 at multiple epochs. July $1996,15 \mathrm{GHz}$ images are presented. It is important to note that we have selfcalibrated our data with the assumption of zero circular polarization $(V)$; this will not create spurious $V$ but may reduce or relocate existing $V$ (Homan et al., in preparation). This may be the case in $3 \mathrm{C} 279$, see figure 2 .

The detection of circular polarization gives us a new probe of the magnetic field structure and particle composition of parsec-scale radio jets. There are two possible sources of circular polarization from synchrotron sources. Intrinsic circular polarization is produced as a small (typically $<1 \%$ ) component of synchrotron radiation. It is sensitive to the energy of the radiating particles and the angle of the magnetic field with the line of sight. Intrinsic circular polarization is reduced by the square root of the number of field reversals along the line of sight but can tell us something about the strength of any uni-directional magnetic field along the jet axis.

The second source of circular polarization is Faraday conversion of linear polarization to circular polarization. This effect requires a population of low energy relativistic particles, and either internal Faraday rotation or a somewhat tangled magnetic field. It is independent of the sign of the charged particles, and works equally well with electrons, positrons, or a mixture of the two. In 3C 279, we believe the circular polarization we observe is the result of Faraday conversion. Coupled with the observed lack of Faraday rotation by the same particles, this suggests that the radiating particles in the jet are a mixture of electrons and positrons (Wardle et al., in preparation).

Acknowledgments. This work has been supported by NSF Grants AST 92-24848 and AST 95-29228, and NASA Grant NGT-51658. The National Radio Astronomy Observatory is a facility of the National Science Foundation, operated under a cooperative agreement by Associated Universities, Inc. 


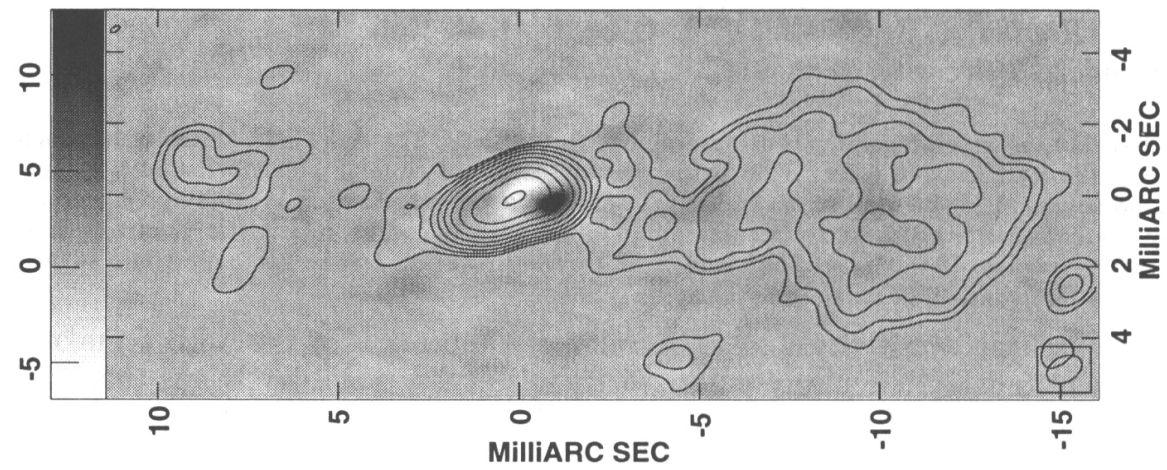

Figure 1. July 1996 images of $3 \mathrm{C} 84$, with total intensity $(I)$ contours (in factors of two ranging from $15 \mathrm{mJy}$ to $3.84 \mathrm{Jy}$ ) superimposed on greyscale circular polarization $(V)$. The $V$ structure lies slightly south and east of the core, and has a peak of $13.3 \mathrm{mJy}$, corresponding to $\simeq 1 \%$ local circular polarization. The smaller negative component closer to the core may be an artifact of our calibration procedure, but could represent the effects of changing opacity through the core.
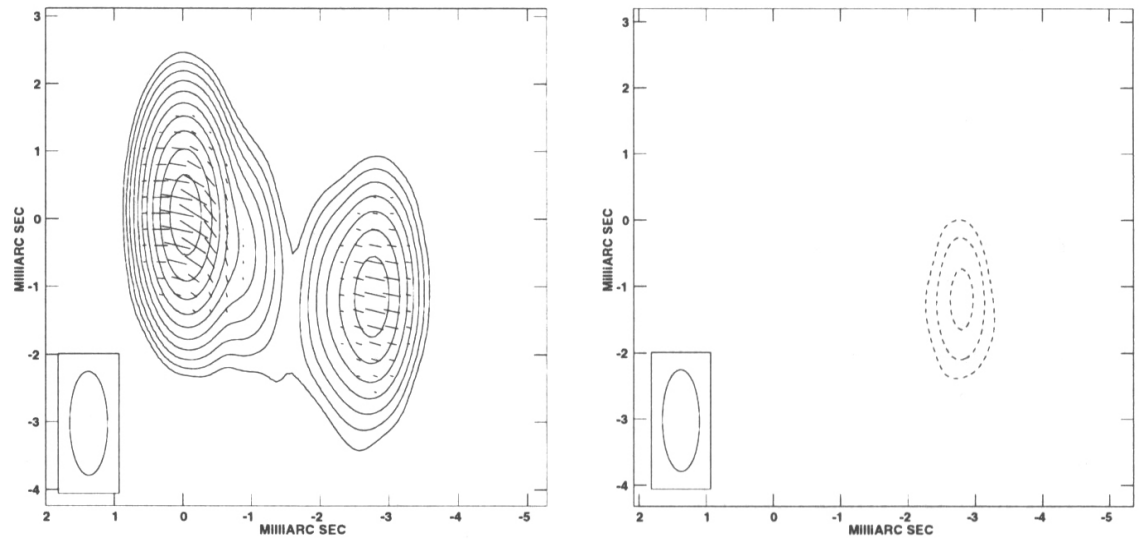

Figure 2. July 1996 images of 3 C 279. (Left) Linear polarization $(P)$ vectors are superimposed on $I$ contours (ranging in factors of two from $15 \mathrm{~m} \mathrm{Jy}$ to $15.36 \mathrm{Jy}$ ). The $I$ structure shows a core-jet morphology with a bright knot in the jet located 3 mas from the core. This knot is linearly polarized at the $10 \%$ level with E-vectors well aligned with the jet axis, suggesting a shock in the jet at this point. (Right) Circular polarization $(V)$ structure with contours at $-2,-4$, and $-8 \mathrm{mJy}$. The $V$ lies right on the bright knot in the jet, and has a peak flux of $-10 \mathrm{mJy}$, corresponding to $-0.5 \%$ local circular polarization. Due to an ambiguity in our calibration technique we can not distinguish this $V$ signal from a $+0.5 \%$ circularly polarized component on the core. 